\title{
Wilms tumor rupture after minimal renal trauma, case report
}

\begin{abstract}
Background: Wilms tumor diagnosis due to minimal abdominal trauma is rare and represents a diagnostic and therapeutic challenge.

Introduction: A 3-year-old girl presented with one week history of slight left flank pain after fell down from her own height, associated with nausea and vomiting. Physical examination showed abdominal bloating and severe left flank pain. The CT scan showed kidney grade III injury and an upper pole solid mass of $12.8 \times 9 \times 7 \mathrm{~cm}$ and $68 \mathrm{UH}$. Nephrectomy was performed. Histopathology reported a Wilms tumor of $13 \times 8 \times 6 \mathrm{~cm}$ with Gerota fascia rupture. Nine months later she came back with pulmonary and peritoneal metastases.
\end{abstract}

Conclusion: Renal trauma is more common in children due to anatomical abnormalities and any discrepancy between the mechanism of injury and physical examination should be suspected for congenital or pathological issues.

Keywords: wilms tumor, abdominal trauma, nephrectomy, pediatric tumors, intestinal obstruction

\author{
Volume 3 Issue I - 2015 \\ Luis Angel Medina Andrade,' Ariel Lozano,' \\ Brenda Ruiz,' Stephanie Serrano Collazos,' \\ Cecilia López,' Israel López,' Pedro \\ Palaciosm, ${ }^{2}$ Luis Cabrera, ${ }^{3}$ Manuel Calao, ${ }^{3}$ \\ Sheila Urbina Hernandez, ${ }^{4}$ José Maria \\ Aguirre Trejo ${ }^{4}$ \\ 'Department of General Surgery, Universidad de Quintana Roo, \\ Mexico \\ ${ }^{2}$ Department of Urology, Instituto Mexicano del Seguro Social \\ ${ }^{3}$ Department of Urology, Universidad de Monterrey, Mexico \\ ${ }^{4}$ Instituto de Estudios Superiores de Chiapas Universidad \\ Salazar, Mexico
}
Correspondence: Luis Angel Medina Andrade, Department of General Surgery, Universidad de Quintana Roo, Instituto Mexicano del Seguro Social, Hospital General Regional No. 17, Servicio de Cirugía General,Av. Politécnico Manzana I Lote I Región 509 C.P. 55750, Cancún, Quintana Roo, Mexico, Tel (+52) (55) 9981963197, (+55) 998। II420।, Email buismedina_5@hotmail.com

Received: October 19,2015 | Published: October 24, 2015
Abbreviations: FAST, focused assessment with sonography in trauma; WT, wilms tumor

\section{Introduction}

The kidney in pediatric patients is more susceptible to further injury by abdominal trauma, consequence of decreased protection mechanisms. Among them are a softer and less extensive rib cage, weaker abdominal muscles, lower amount of perirenal fat and lower abdominal position than adults. ${ }^{1,2}$ Anatomical abnormalities have a higher incidence in pediatric patients without association with severity of clinical manifestations by trauma. Clinical signs of severe renal trauma like hematuria have a low frequency of occurrence in children. Hypotension is not a suitable parameter to assess bleeding since there is an increase in sympathetic tone that allows normal blood pressure in pediatric population. ${ }^{3}$ The decision of surgical exploration in these patients has to be made based on imaging studies, laboratories and clinical findings. Focused Assessment with Sonography in Trauma (FAST) sensitivity ranges from 22 to $85 \%$ depending on the operator. The abdomino-pelvic triphasic computed tomography is always preferred when the patient is hemodynamically stable for its sensitivity near to $98 \%$. Treatment of kidney injury should be guided by injury degree, clinical findings and hemodynamic stability. Currently conservative management is preferred in grade I to IV, consisting of continuous monitoring and subsequent extension studies to determine the extent of kidney injury.,

Nephroblastoma or Wilms tumor (WT) represents $90 \%$ of renal tumors in children and treatment is a surgical emergency so it is responsible for $5 \%$ of pediatric malignant tumors. ${ }^{6} \mathrm{WT}$ is usually asymptomatic until a solid abdominal mass is detected. It has been shown that the use of preoperative chemotherapy and radiotherapy for large tumors and other structures decreases recurrence. In case of spontaneous tumor rupture, there is staging change from I to III, with tumor cells embolization and local extension. ${ }^{7}$

\section{Case presentation}

We report the case of a 3-year-old female, with no past medical history. She was admitted to the emergency room after one week left flank pain associated with nausea and vomiting for the last 24 hours. Her mother reports that a week before she tripped hitting the floor in the left upper quadrant, with minimal pain in this area until the last 24hours. Physical examination did not evidence hematoma or skin excoriations; we found abdominal bloating, lack of peristalsis and severe pain on palpation in the left flank. Laboratory tests were performed reporting no microscopic hematuria, creatinine $0.8 \mathrm{mg} / \mathrm{dl}$, hemoglobin $6 \mathrm{mg} / \mathrm{dL}$ and hematocrit of $19 \%$. Abdominal computed tomography: left renal hematoma that does not allow the display of spleen, with lower extension and displacement of bowel loops, with no involvement of renal hilum (Figure 1A). Within the hematoma, a $12.8 \times 9 \times 7 \mathrm{~cm}$ solid lesion with heterogeneous areas located up to 68 UH was identified (Figure 1B). The patient was hemodynamically stable but with persistence of pain and intestinal obstruction secondary to hematoma compression. Clinical presentation with signs and symptoms not according to trauma mechanism and CT scan reports made us suspect the presence of a tumor in the affected kidney. Therefore abdominal exploration was performed; renal hematoma and grade III kidney injury with rupture tumor were found. Left nephrectomy is decided at that time (Figure 2A). 
After transfusion of 3 red blood cell packs and $72 \mathrm{~h}$ of observation patient was sent home. The histopathological report was of a Stage III Wilms tumor with Gerota fascia rupture without nodal or vascular disease (Figure 2B). Blastemal component $60 \%$, epithelial component $40 \%$, necrosis $10 \%$ and no evidence of anaplastic were reported (Figure 3). The patient was lost to follow up until four months later when she was asymptomatic and with normal renal function at this moment. She was sent to pediatric oncology but did not attend follow until 9months later when she came back to emergency room due to respiratory distress associated with respiratory tract infection. The CT scan reported pulmonary and abdominal metastasis. The patient received standard chemotherapy treatment in other institution.

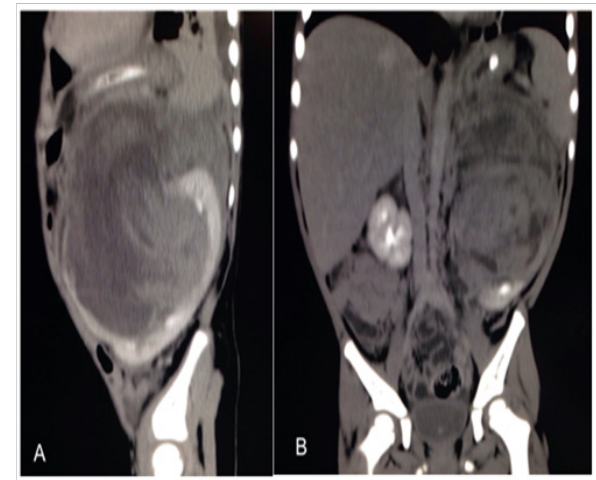

Figure I A) TAC with left renal hematoma with lower extension and displacement of bowel loops, without involvement of the renal hilum.

B) Coronal view with a left kidney heterogeneous lesion under the hematoma.

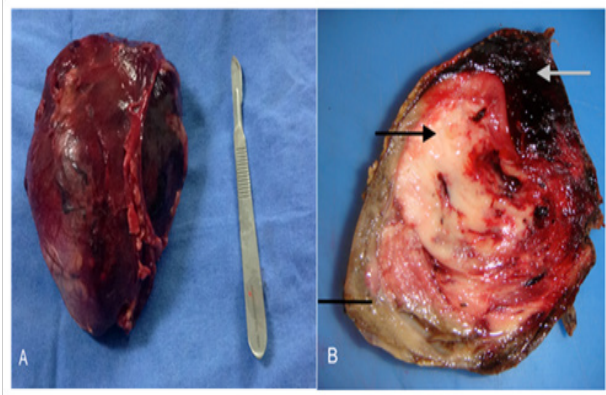

Figure 2 A) Left Kidney in the post operatory.

B) Left kidney coronal cut, illustrates hematoma (white arrow), tumor (black arrow) and residual kidney (Black line).

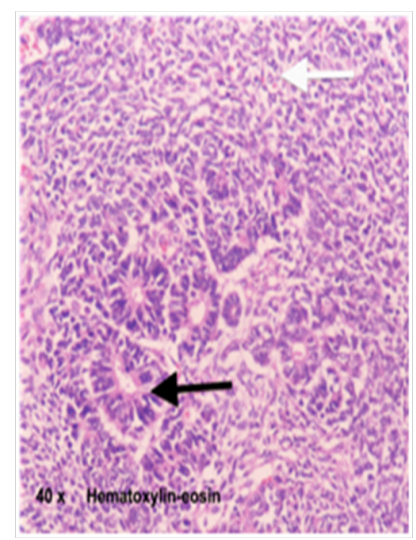

Figure $340 x$ hematoxylin-eosin microscopy of tumor with $60 \%$ blastemal component (white arrow), $40 \%$ epithelial component (black arrow) and no evidence of anaplastic cells.

\section{Discussion}

Currently the renal trauma can be managed conservatively in almost all cases as long as the patient remains hemodynamically stable without significant clinical changes. Continuous monitoring and serial imaging studies are performed because of the presence of extensive hematoma, renal dissociated fragments, interpolate contrast extravasation $>=4 \mathrm{~cm}$ urinoma and intense pain or fever are associated with conservative treatment fails and surgery. ${ }^{8-10}$ Surgical exploration is not indicated from the beginning since this entails a higher index of nephrectomy. ${ }^{10}$ The congenital abnormalities and tumors have a higher incidence in patients with abdominal trauma in pediatric age, with an incidence of up to $12.6 \%$. In rare cases initial presentation is secondary to minimal trauma. Therefore it can be difficult to diagnose and shall be highly suspected when the mechanism of injury is not consistent with the magnitude or persistence of symptoms. ${ }^{10}$

Wilms tumor is the most common children renal tumor. It course asymptomatic until a large abdominal mass is detected or minimal intestinal disorders are developed. Once diagnostic is made, total tumor resection must be completed urgently. In case of local or vascular spread, resection must be performed after neoadjuvant therapy, allowing no recurrence in up to $95 \%$ of cases. Wilms tumor rupture changes the stage I to III, which worsens the prognosis, rising recurrence index to $16.2 \%$ in big pediatric centers. ${ }^{7}$ The association of severe renal injury with low energy transmission is very rare, favored by the presence of congenital abnormalities or a tumor. In this case the intervention of the patient was urgently due to abdominal complications and imaging findings, with a high suspicion of tumor lesion. The presentation of increasing abdominal pain could be secondary to rebleeding after initial formed clot reabsorption since the $5^{\text {th }}$ day after trauma. ${ }^{3}$

Such patients should be referred to a specialized pediatric surgery center, as they have reported better results with a lower rate of recurrence. Because the presentation of this case with traumatic rupture of the tumor and its assessment a week later, prognosis was less favorable after appropriate treatment, favoring the subsequent relapse, having to receive second-line chemotherapy and radiotherapy.

\section{Acknowledgements}

Claudia Cavazos $\mathrm{PhD}$, for her contribution to the pathology photographs and analyses.

\section{Conflict of interest}

The author declares no conflict of interest.

\section{References}

1. Stepan JG, Gelberman RH, Rubin DA, et al. Extra-articular lipoma arborescens of the dorsal aspect of the wrist with invasion of the extensor tendons a case report. JBJS Case Connect. 2013;3(1):e30.

2. Brown SL, Elder JS, Spirnak JP. Are pediatric patients more susceptible to major renal injury from blunt trauma? A comparative study. $J$ Urol. 1998;160(1):138-140.

3. Bschleipfer T, Kallieris D, Hauck EW, et al. Blunt renal trauma: biomechanics and origination of renal lesions. Eur Urol. 2002;42(6):614-621.

4. Husmann Douglas. Pediatric Genitourinary Trauma. In: Campbell editor. Pedaitric Urology. 1st ed. Saunders, USA: EUA; 2014. p. 3731-3753. 
5. Partrick DA, Bensard DD, Janik JS, et al. Is hypotension a reliable indicator of blood loss from traumatic injury in children? Am J Surg. 2002;184(6):559-560

6. Dalrymple Neal, Oliphant Michael. Imaging evaluation of trauma. Elsevier. 2014;9:184-214.

7. Friedman AD. Wilms Tumor. Pediatrics in Review. 2013;34(7):328330.

8. Huddart Simon. Wilms tumour-the surgical issues. Paediatrics and child health. 2013;24(4):137-142.
9. Reese JN, Fox JA, Cannon GM, et al. Timing and predictors for urinary drainage in children with expectantly managed grade iv renal trauma. $J$ Urol. 2014;192(2):512-518.

10. Yang Chia-Shen, Chen Isaac, Wang Chien, et al. Predictive indications of operation and mortality following renal trauma. J Chin Med Assoc. 2012;75(1):21-24.

11. Salem Hosni, Morsi Hani, Zakaria Ahmad. Management of high grade renal injuries in children after blunt abdominal trauma:Experience of 40 cases. J Ped Urol. 2007;3(3):223-229. 